\title{
Optical and tomography studies of water- soluble gold nanoparticles on bacterial exopolysaccharides
}

Cite as: J. Appl. Phys. 126, 053101 (2019); https://doi.org/10.1063/1.5090879

Submitted: 30 January 2019 . Accepted: 16 June 2019. Published Online: 01 August 2019

Ana González (D), Víctor Garcés, Laura Sabio (D), Felix Velando, Miguel López-Haro, Natividad Gálvez, Jose

J. Calvino, and Jose M. Dominguez-Vera (D)

\section{COLLECTIONS}

Paper published as part of the special topic on Magnetic and Plasmonic Nanoparticles for Biomedical Devices Note: This paper is part of the Special Topic on Magnetic and Plasmonic Nanoparticles for Biomedical Devices.

F This paper was selected as Featured
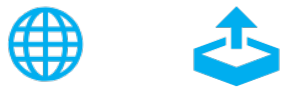

\section{ARTICLES YOU MAY BE INTERESTED IN}

Visualizing and monitoring interface structures and dynamics by luminogens with aggregation-induced emission

Journal of Applied Physics 126, 050901 (2019); https://doi.org/10.1063/1.5110688

Perspective on ab initio phonon thermal transport

Journal of Applied Physics 126, 050902 (2019); https://doi.org/10.1063/1.5108651

Straintronics in graphene: Extra large electronic band gap induced by tensile and shear strains Journal of Applied Physics 126, 054302 (2019); https://doi.org/10.1063/1.5095600

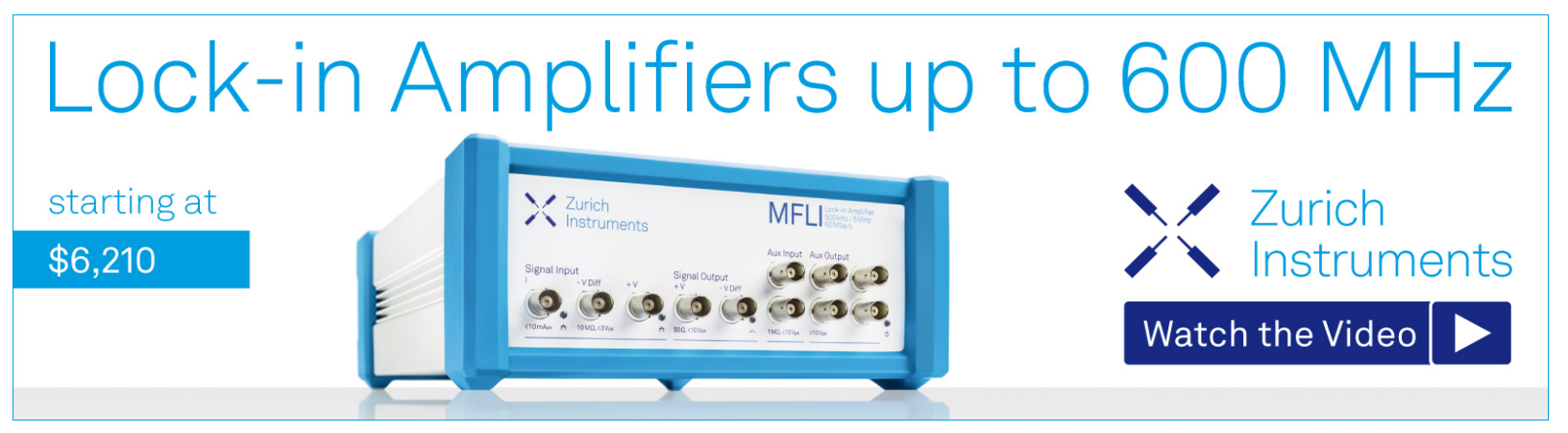




\title{
Optical and tomography studies of water-soluble gold nanoparticles on bacterial exopolysaccharides
}

\author{
Cite as: J. Appl. Phys. 126, 053101 (2019); doi: 10.1063/1.5090879 \\ Submitted: 30 January 2019 . Accepted: 16 June 2019 . \\ Published Online: 1 August 2019
}

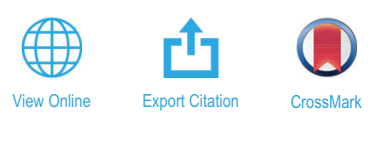

Ana González, ${ }^{1}$ (D Víctor Garcés, ${ }^{7}$ Laura Sabio, ${ }^{1}$ (D) Felix Velando,, ${ }^{7}$ Miguel López-Haro, ${ }^{2}$ Natividad Gálvez, Jose J. Calvino, ${ }^{2, a)}$ and Jose M. Dominguez-Vera ${ }^{1, a)}$ (D)

\begin{abstract}
AFFILIATIONS
${ }^{1}$ Departamento de Química Inorgánica and Instituto de Biotecnología, Universidad de Granada, 18071 Granada, Spain

${ }^{2}$ Departamento Ciencia de Materiales e Ingeniería Metalúrgica y Química Inorgánica, Universidad de Cádiz, Campus Río San

Pedro, 11510 Cádiz, Spain
\end{abstract}

Note: This paper is part of the Special Topic on Magnetic and Plasmonic Nanoparticles for Biomedical Devices.

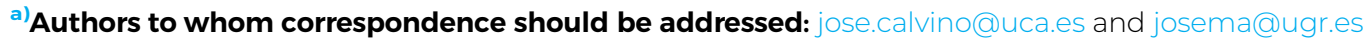

\begin{abstract}
Gold nanoparticles of different shapes (spherical, rods, and prisms) aggregate when deposited onto Lactobacillus fermentum's exopolysaccharide (EPS), a set of polysaccharides excreted by the bacteria. Transmission electron microscopy studies revealed that gold nanoparticles have high affinity for EPS. UV-vis spectra of aggregated gold nanoparticles showed additional absorbance peaks at lower energies in comparison with isolated nanoparticles. In the case of gold nanoprisms, the aggregation leads to a new absorption at a very low energy centered at $1100 \mathrm{~nm}$. Moreover, the EPS of L. fermentum itself produces gold aggregates from a Au(III) solution. Surface-enhanced Raman spectroscopy performances for the detection of rhodamine B of gold aggregates were drastically different. A tomography study on all samples revealed clear differences in the extension of the EPS coating on the gold nanoparticles. Only the gold aggregate in which gold interparticle surfaces were exposed to $\mathrm{RhB}$ showed a drastic increase (two orders of magnitude) of intensity in the Raman spectrum of RhB.
\end{abstract}

Published under license by AIP Publishing. https://doi.org/10.1063/1.5090879

\section{INTRODUCTION}

Gold nanoparticles (AuNPs) have attracted considerable attention because of their extraordinary properties, especially optical properties. ${ }^{1-4}$ Furthermore, gold is one of few metals that survive as nanoparticles under atmospheric conditions. The combination of optical properties and stability in aerobic environments has enabled AuNPs to become one of the most important materials in nanotechnology. ${ }^{5-10}$

AuNPs exhibit a strong absorption band in the visible region, due to a small particle effect absent in individual atoms and bulk materials. This absorption results when the incident photon frequency is resonant with the collective oscillation of the conduction band electrons (the so-called plasmons). ${ }^{1-4}$ The phenomenon is known as surface plasmon resonance (SPR). SPR is the key to optical properties of AuNPs and is strongly dependent upon the size, shape, chemical environment, and aggregation of nanoparticles. ${ }^{1-4}$ Fine-tuning these parameters enables the production of AuNPs with desired optical properties for specific applications.

Spherical gold nanoparticles (AuNSs) have usually a SPR centered in the range of $500-600 \mathrm{~nm}$. The absorptions at these wavelengths limit the use of AuNSs in biomedicine since blood and soft tissues absorb at these wavelengths. However, when SPR of AuNPs occurs at lower energies such as the near-infrared (NIR), the clinical use of AuNPs can extend beyond superficial applications, since NIR light can penetrate much deeper to reach AuNPs inside the body.

Two frequent approaches to shift the SPR of AuNPs to lower energies are the production of anisotropically-shaped AuNPs ${ }^{11}$ and the assembly of nanoparticles. ${ }^{12}$ The first method of shifting AuNP SPR involves synthesizing nanoparticles with anisotropic geometries. In gold nanorods (AuNRs), the SPR splits into two bands: the transverse mode shows a resonance at about $500 \mathrm{~nm}$, which is co-incident with the SPR of spherical particles, while the resonance of the longitudinal mode is red-shifted to wavelengths depending on the length to width ratio of the nanorods. In shapes with even greater anisotropy, such as gold nanoprisms (AuNPrs), the lowenergy SPR falls in the near-infrared region with wavelengths reaching values of $1100 \mathrm{~nm}$, whereas the transverse mode also appears at about $500 \mathrm{~nm}$. 
A second approach to shifting AuNP SPR to NIR wavelengths is using the formation of nanoparticle aggregates. The SPR changes drastically if particles are densely packed such that individual particles are electronically coupled to each other. The SPR of the aggregate is red-shifted depending on coordination number and interparticle distances. ${ }^{12}$

The SPR shift is not the only consequence produced by aggregation of AuNPs. In comparison to isolated nanoparticles, AuNP aggregates increase the surface-enhanced Raman spectroscopy (SERS) effect due to the formation of hot spot in the interparticle region. AuNPs act as antennas for incident light with the same frequency as the collective oscillation of plasmons. The AuNPs, therefore, concentrate the oscillating electric fields at their surfaces, with the same frequency as the incident light but with several orders of magnitude of higher intensities. The electric field enhancement excites the Raman modes of the molecule being studied, therefore increasing the signal of the Raman scattering. ${ }^{13-17}$ SERS is currently one of the most powerful spectroscopic methods for ultrasensitive detection of molecules.

Therefore, aggregation can both improve optical properties and lead to additional applications. With this in mind, different approaches to synthesizing AuNP aggregates have arisen: hydrogen bonding, electrostatic forces, layer-by-layer techniques, substrates template, etc. ${ }^{1-4}$ In the present work, AuNP aggregates were produced using exopolysaccharides (EPSs) of Lactobacillus fermentum, a probiotic that constitutes an important part of the natural healthy microbiota.

EPSs are natural polysaccharides secreted by some bacteria. They play a crucial role in bacterial surface adhesion, an essential step for colonization. ${ }^{18}$ In particular, L. fermentum's EPS allows colonization of these healthy bacteria in intestines by facilitating adhesion to enterocyte cells. Furthermore, L. fermentum's EPS is digestible, biocompatible, and approved by governmental health agencies (e.g., Food and Drug Administration). 19,20

We produced AuNP aggregates using EPS of L. fermentum with two different approaches: (i) EPS as a surface for deposition of previously prepared AuNPs with defined size and shape and (ii) EPS as a combined reducing and stabilizing agent. The resulting AuNP aggregates were soluble in water and differ in optical properties and SERS effects. To rationalize the huge differences found in SERS effects, we carried out an electronic microscopy tomography study on the nanostructures. These studies allowed us to conclude that improving optical properties and SERS effects largely depends on the access of the analyte to the hot spot generated by gold nanoparticles aggregation.

\section{METHODS}

\section{A. Materials}

All reagents were purchased from Sigma Aldrich. UV-vis spectra were recorded at a Unicam UV 300 Thermo Spectronic spectrophotometer.

\section{B. Bacterial growth}

L. fermentum was grown in anaerobic conditions in a synthetic growth medium at $37^{\circ} \mathrm{C}$ on an orbital shaker for $24 \mathrm{~h}$ with an initial concentration of $1 \mathrm{mg}$ bacteria in $1 \mathrm{ml}$ of medium. The synthetic growth medium consisted of $\left(\mathrm{gl}^{-1}\right) \mathrm{Na}_{2} \mathrm{HPO}_{4}-5.0, \mathrm{KH}_{2} \mathrm{PO}_{4}-6.0$, trisammonium citrate-2.0, sucrose $-50.0, \mathrm{MgSO}_{4}-1.0$, and trace elements solution-10 $\mathrm{ml}$ [consisting of $\left(\mathrm{gl}^{-1}\right): \mathrm{MnSO}_{4}-2.0$, $\mathrm{CoCl}_{2}-1.0, \mathrm{ZnCl}_{2}-1.0$ dissolved in $0.1 \mathrm{~N} \mathrm{HCl}$ solution]. The medium had an initial $\mathrm{pH}$ of 6.7 and was sterilized at $121^{\circ} \mathrm{C}$. The final L. fermentum cell concentration was $3.3 \cdot 10^{8} \mathrm{CFU} \mathrm{ml}^{-1}$.

\section{EPS of L. fermentum isolation}

EPS of L. fermentum were isolated following a previous reported protocol. ${ }^{21}$ Bacteria were removed by centrifugation at $3000 \mathrm{~g}$ for $10 \mathrm{~min}$. The supernatant was filtered using EMD Millipore Steritop ${ }^{\mathrm{TM}}$ Sterile Vacuum Bottle-Top Filters. Ice-cold ethanol ( 2 vol.) was added to the cell-free supernatant ( $1 \mathrm{vol}$.) under continuous stirring and kept overnight for precipitation in the refrigerator. The alcoholic supernatant was centrifuged at $22000 \mathrm{~g}$ for $35 \mathrm{~min}$ and the EPS precipitated were washed with acetone and again centrifuged. The solid obtained was dissolved in water $(20 \mathrm{ml})$, filtered again, and lyophilized.

\section{Synthesis of spherical gold nanoparticles (AuNSs)}

AuNSs were synthesized using a protocol previously reported. ${ }^{22} 5 \mathrm{ml}$ of a $1.0 \mathrm{mM} \mathrm{HAuCl}_{4}$ solution in water was stirred and heated to boiling on a hot plate. After the solution began to boil, $500 \mu \mathrm{l}$ of a $38.8 \mathrm{mM} \mathrm{Na}_{3} \mathrm{C}_{6} \mathrm{H}_{5} \mathrm{O}_{7}$ solution in water was added. The mixture was boiled and stirred continuously for about $10 \mathrm{~min}$ until it was a deep red color. The solution was cooled to room temperature. The AuNSs were characterized by UV-vis spectroscopy and transmission electron microscopy (TEM).

\section{E. Synthesis of gold nanorods (AuNRs)}

To prepare AuNRs, a seed-mediated growth method was carried out. ${ }^{23}$ Solutions of $\mathrm{HAuCl}_{4} 1 \mathrm{mM}, \mathrm{AgNO}_{3} 8 \mathrm{mM}$, ascorbic acid $78.8 \mathrm{mM}$, and $\mathrm{NaBH}_{4} 10 \mathrm{mM}$ were prepared at room temperature. The $\mathrm{NaBH}_{4}$ solution was ice-cooled after preparation. Solutions of cetyl trimethylammonium bromide (CTAB) $0.2 \mathrm{M}$ and (11-mercaptoundecyl)-N,N,N-trimethylammonium bromide $92 \mathrm{mM}$ were separately prepared by heating at $50{ }^{\circ} \mathrm{C}$ and stirring until dissolved and then cooled to room temperature. All the solutions were prepared in Milli-Q ultrapure water. Gold seed synthesis: $5 \mathrm{ml}$ of CTAB $0.2 \mathrm{M}, 2.5 \mathrm{ml} \mathrm{HAuCl}_{4} 1 \mathrm{mM}$, and $0.6 \mathrm{ml}$ of ice-cold $\mathrm{NaBH}_{4}$ $10 \mathrm{mM}$ were mixed and stirred for $2 \mathrm{~min}$ at $25^{\circ} \mathrm{C}$. When seeds were formed, the solution color changed from yellow to slightly brown. NRs synthesis: $5 \mathrm{ml}$ of the $0.2 \mathrm{M}$ CTAB solution was placed in a flask previously set in an oil bath at $30^{\circ} \mathrm{C}$ under stirring at $180 \mathrm{rpm} .5 \mathrm{ml}$ of $\mathrm{HAuCl}_{4} 1 \mathrm{mM}, 70 \mu \mathrm{l}$ of ascorbic acid $78.8 \mathrm{mM}$, and $100 \mu \mathrm{l}$ of $\mathrm{AgNO}_{3} 8 \mathrm{mM}$ were added in this order to the CTAB solution. The mixture was completed with $160 \mu \mathrm{l}$ of the seed solution. The completed mixture was kept stirring at $180 \mathrm{rpm}$ and $30^{\circ} \mathrm{C}$ for $48 \mathrm{~h}$. Due to $\mathrm{CTAB}$ antibacterial activity, ${ }^{24} \mathrm{CTAB}$ excess was removed. AuNRs were collected twice by high-speed centrifugation at $13000 \mathrm{rpm}$ for $10 \mathrm{~min}$, and the supernatant was removed and the collected nanorods were dissolved in $1 \mathrm{ml}$ of Milli-Q ultrapure water and $1 \mathrm{ml}$ of (11-mercaptoundecyl)-N,N,N-trimethylammonium bromide $92 \mathrm{mM}$ and stirred mildly. AuNRs were collected again by high-speed centrifugation at $13000 \mathrm{rpm}$ for $10 \mathrm{~min} 48 \mathrm{~h}$ later, and the pellet was dissolved in $1 \mathrm{ml}$ of Milli-Q ultrapure water. The 
AuNRs were characterized by UV-vis spectroscopy and transmission electron microscopy (TEM).

\section{F. Synthesis of gold nanoprisms (AuNPrs)}

AuNPrs were synthesized using a protocol previously reported. ${ }^{25} 100 \mathrm{ml}$ of $\mathrm{HAuCl}_{4} 2 \mathrm{mM}$ and $120 \mathrm{ml}$ of fresh $\mathrm{Na}_{2} \mathrm{~S}_{2} \mathrm{O}_{3}$ $0.5 \mathrm{mM}$, both prepared in Milli-Q ultrapure water, were mixed and stirred gently at $15^{\circ} \mathrm{C}$. After 9 min ("seed" formation), an extra $50 \mathrm{ml}$ of fresh $\mathrm{Na}_{2} \mathrm{~S}_{2} \mathrm{O}_{3} 0.5 \mathrm{mM}$ was added. Growth mixture was left overnight at $15^{\circ} \mathrm{C}$ under mild stirring conditions. The AuNPrs were characterized by UV-vis spectroscopy and TEM.

\section{G. Deposition of AuNPs onto EPS}

A $10 \mathrm{mg} \mathrm{ml}^{-1}$ EPS solution in water was adjusted to $\mathrm{pH} 2$ using $\mathrm{HCl} 1 \mathrm{M}$. The EPS solution was heated for $24 \mathrm{~h}$ at $80^{\circ} \mathrm{C}$ under continuous stirring at $200 \mathrm{rpm}$. The solution was cooled to room temperature. AuNSs, AuNRs, and AuNPrs (1 vol.) were added to three different aliquots of the cooled EPS pH2 solution (1 vol.). The Au nanoparticle aggregation onto EPS was characterized by UV-vis spectroscopy, TEM, and energy dispersive X-ray spectroscopy (EDX) compositional maps collected with High Annular Dark Field-Scanning Transmission Electron Microscopy (HAADF-STEM) images. All EPS-samples were lyophilized and stored as powders. Redissolution of the powders in water gave unchanged UV-vis spectra.

\section{H. Synthesis of gold nanoparticles using EPS as reductant (Au-EPS)}

A $2.5 \mathrm{ml} 1.0 \mathrm{mM} \mathrm{HAuCl}$ solution was stirred and heated to boiling on a stir/hot plate. After the solution began to boil, $2.5 \mathrm{ml}$ of a $10 \mathrm{mg} \mathrm{ml}^{-1}$ EPS aqueous solution ( $\mathrm{pH} \mathrm{2)}$ was added. The mixture was boiled and stirred for $30 \mathrm{~min}$ until it was a purple/blue color and then cooled to room temperature. The samples were characterized by UV-vis spectroscopy, TEM, and EDX compositional maps collected with HAADF-STEM images.

\section{Electronic microscopy sample preparation}

TEM samples were prepared by placing a drop of each sample onto a FCF200-Cu (Formvar/Carbon 200 Mesh, Copper) grid and blotting with filter paper. Samples were observed under a LIBRA 120 PLUS from Carl Zeiss SMT and EDX maps were done using a HAADF-STEM system.

\section{J. SERS experiments}

A $10 \mu \mathrm{M}$ solution of $\mathrm{RhB}$ in water was prepared. A $5 \mu \mathrm{l}$ drop of this solution was added slowly and without stirring to three different solutions each containing $25 \mu \mathrm{l}$ of the isolated gold nanoparticles (AuNSs, AuNRs, and AuNPrs) and $25 \mu \mathrm{l}$ of $\mathrm{H}_{2} \mathrm{O}$. The same volume of $\mathrm{RhB} 10 \mu \mathrm{M}$ was added to $50 \mu \mathrm{l}$ of the AuNSs, AuNRs, and AuNPrs onto EPS samples and to $50 \mu$ l of AuNPs synthesized by EPS at $\mathrm{pH} 2$. The final concentration of $\mathrm{RhB}$ in all samples was $0.5 \mu \mathrm{M}$.

Gold nanoparticles on EPS samples were prepared as described above. An aluminum adhesive was fixed on stubs of metal. After several minutes, a $20 \mu \mathrm{l}$ drop of each sample was placed over a stub and dried overnight at room temperature. Samples were observed under a Zeiss SUPRA40VP microscope and Raman spectra were collected using a Structural Chemical Analyzer (SCA) with excitation at $532 \mathrm{~nm}$ (intensity of $35 \%$ and 3 acquisitions). A study on the stability of the SERS effect was carried out by incubation of gold aggregates and $\mathrm{RhB}$ for different times ( $30 \mathrm{~min}, 1$ and $4 \mathrm{~h}$ ). The intensity of Raman signals was practically the same, pointing out a permanent SERS effect of the gold nanostructures on the RhB.

\section{K. Electron tomography (ET)}

Electron tomography (ET) experiments were performed in a FEI Titan3 Themis 60-300 Double Aberration Corrected microscope operated at $80 \mathrm{kV}$. A convergence angle of $9 \mathrm{mrad}$ was selected in order to improve the depth of focus. HAADF and DF detectors were used to image the EPS and nanoparticles signals, respectively. In particular, a camera length of $185 \mathrm{~mm}$ was used for AuNSs and AuNPrs-EPS and $56 \mathrm{~mm}$ for Au-EPS. Then, a series of STEM-HAADF/DF images at different tilts were recorded using the software FEI Explore3D v.4.1. The tracking, focusing, and tilting were carried out automatically. The samples were tilted from $-70^{\circ}$ to $+70^{\circ}$ and images acquired every $5^{\circ}$. Then, the whole set of images were aligned combining cross-correlation method, using FEI Inspect3D, and the landmark-based alignment implemented in TomoJ. The tilt series images were also background-subtracted, normalized, and binned to $512 \times 512$ pixels. Afterward, they were reconstructed into a 3D volume using a Compressed Sensing algorithm based on Minimization of the Total Variation (TVM) of the individual STEM-HAADF images. In particular, a 3D implementation of the TVAL3 routine, using AstraToolBox, was employed. For visualization and further nanometrological analysis of the reconstructed volumes, the FEI Avizo Software was used. The resolution in electron tomography is anisotropic. In the XY direction, which is that of the projections, the scale bars of the figures are real. In the $\mathrm{XZ}$ direction, the resolution depends on the number of projections and the reconstruction algorithm used. In this work, the advanced algorithm used eliminates elongation artifacts, and the spatial resolution is below $1 \mathrm{~nm}$.

\section{RESULTS AND DISCUSSION}

\section{A. Deposition of AuNPs onto EPS}

First, following well-documented procedures, we prepared AuNPs with the three more representative shapes: spheres (AuNSs), ${ }^{22}$ rods (AuNRs), ${ }^{23,24}$ and prisms (AuNPrs). ${ }^{25}$ The expected shapes, sizes, and SPR values were confirmed by Transmission Electron Microscopy (TEM) and UV-vis spectroscopy (Fig. 1). Next, we incubated gold samples with EPS isolated from L. fermentum ${ }^{21}$ to produce AuNSs-EPS, AuNRs-EPS, and AuNPrs-EPS. In the three samples, large aggregates of nanoparticles on the EPS were revealed by TEM (Fig. 1). The deposition of AuNPs did not result in any changes of shape or size, which is an advantage since other coating often results in reshaping the particles. Moreover, the existence of EPS supporting the gold aggregates makes them water-soluble, which is of crucial interest in many applications.

The aggregates were also examined using High Annular Dark Field-Scanning Transmission Electron Microscopy (HAADF-STEM) 

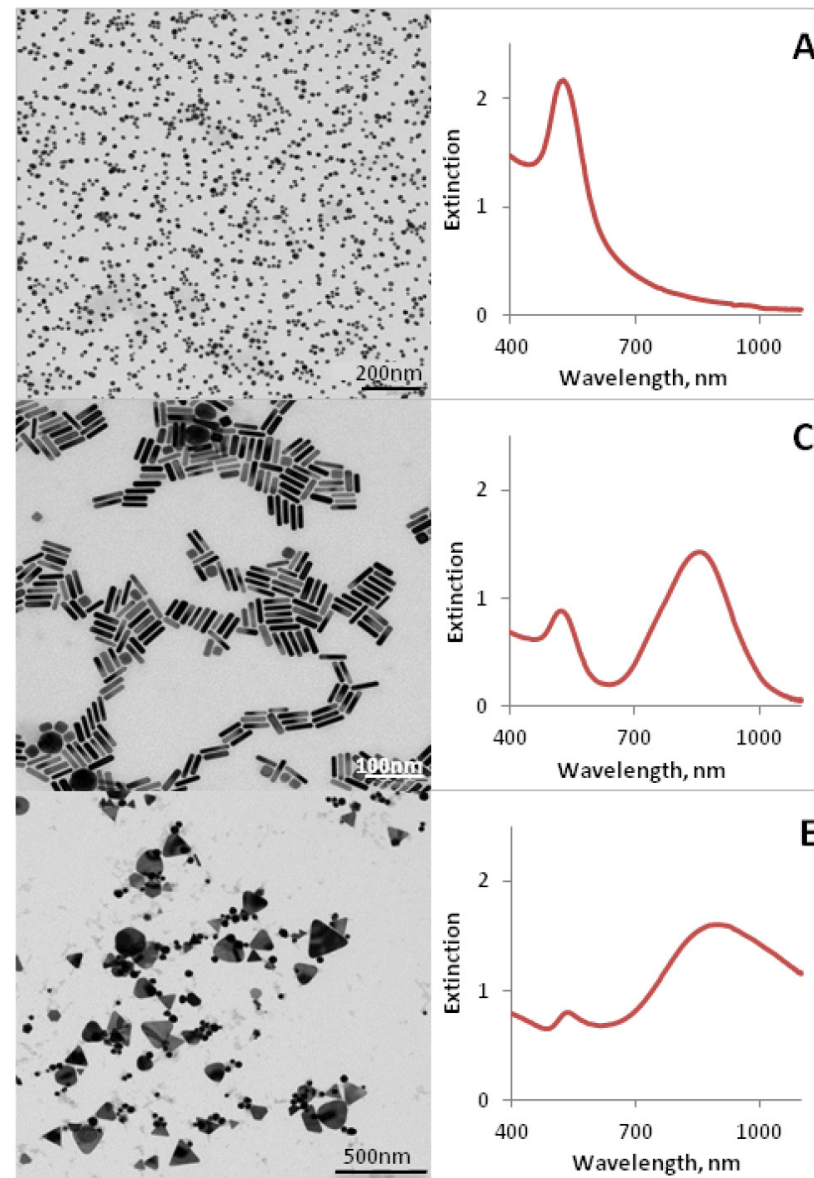

A
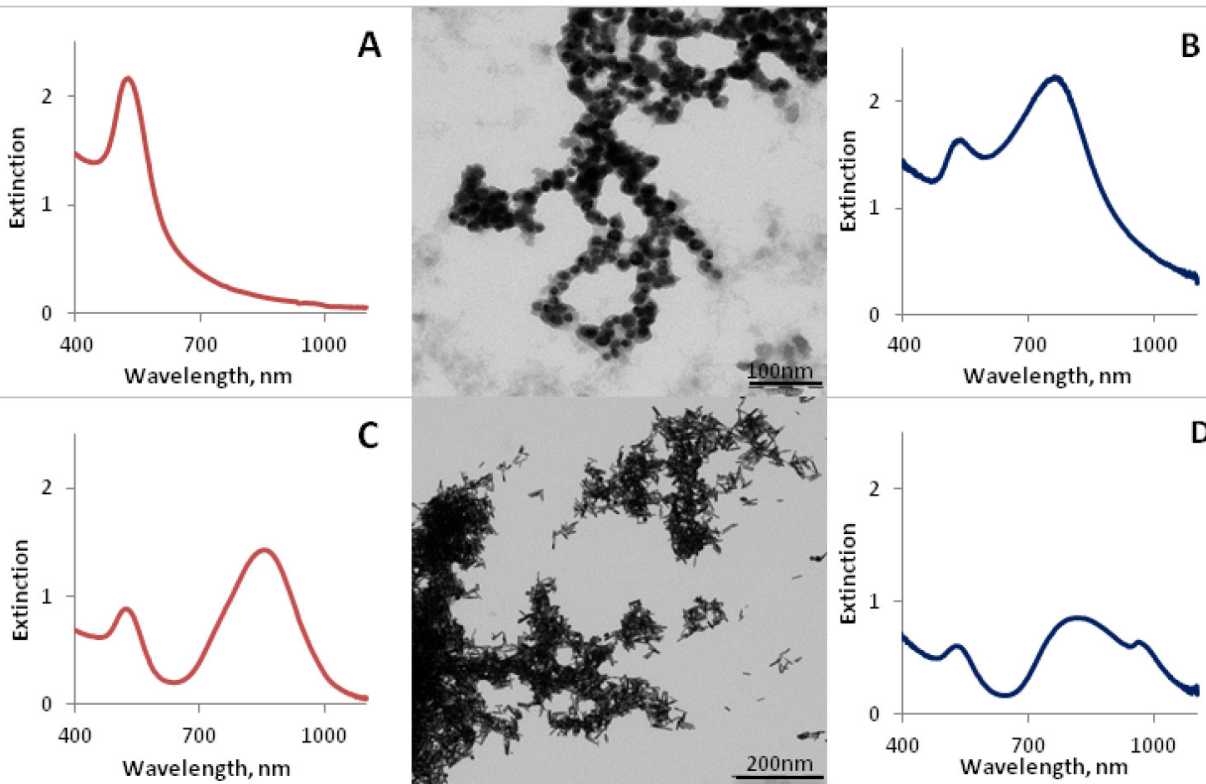

C
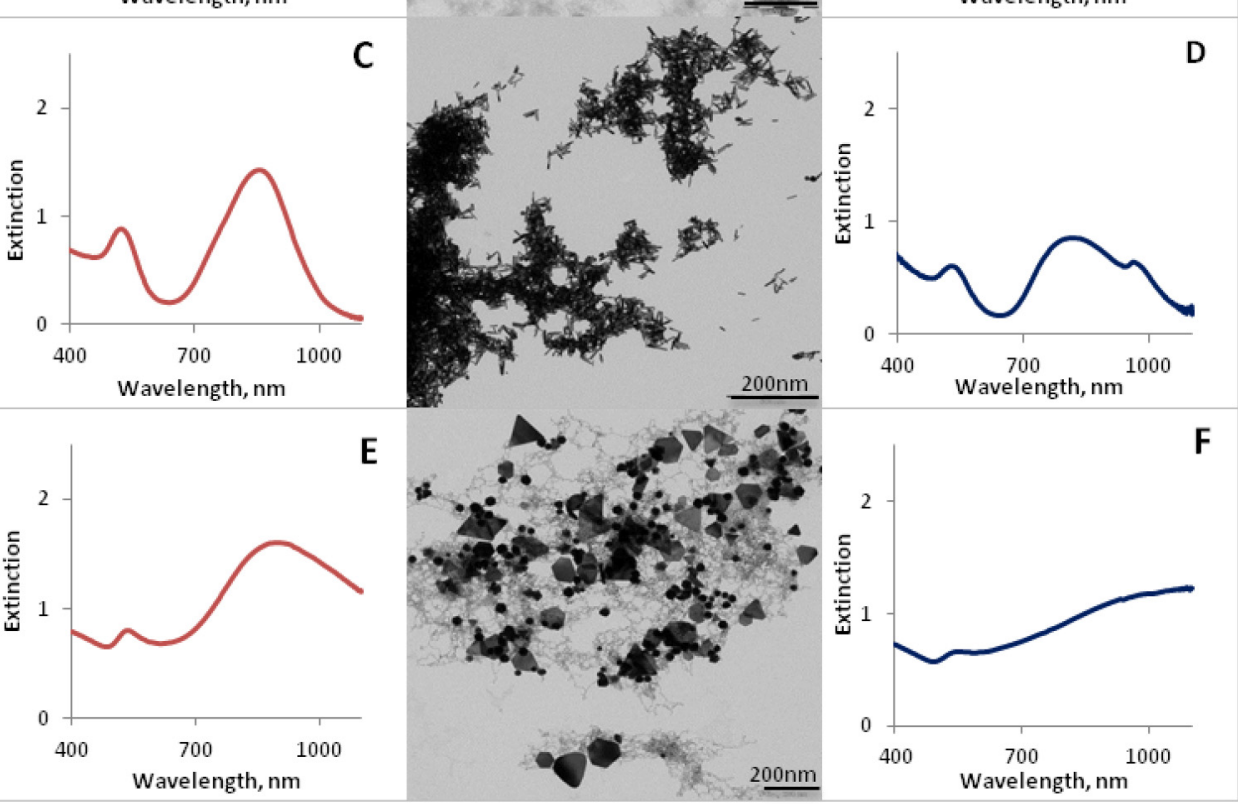

E
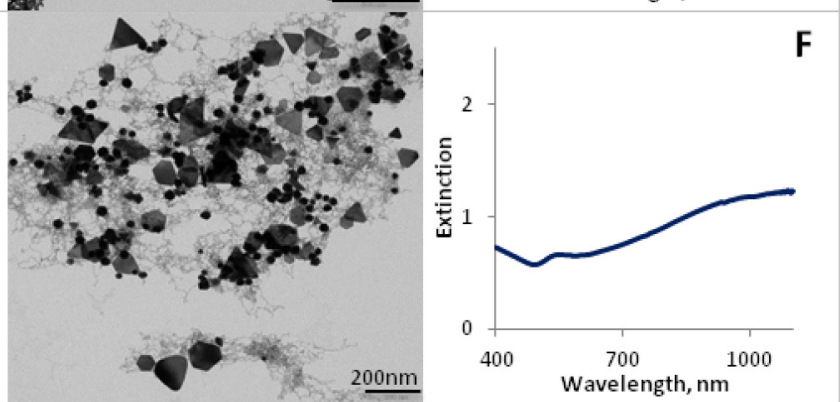

FIG. 1. TEM images and UV-vis spectra of AuNSs, AuNRs, and AuNPrs before [(a), (c), and (e)] and after incubation with EPS to give AuNSs-EPS, AuNRs-EPS, and AuNPrs-EPS [(b), (d), and (f)].

(Fig. 2). To confirm the presence of the AuNPs on the EPS, energy dispersive X-ray spectroscopy (EDX) experiments were performed, which showed that $\mathrm{Au}$ (in violet) is associated to EPS. The spatial distribution of gold, which was barely detectable outside the EPS region, indicates that the AuNPs were glued to the EPS.

As shown in Fig. 1, once the AuNPs were deposited onto EPS, their UV-vis spectra showed additional absorbance peaks at lower energies, which indicate that the particles were assembled into aggregates with strong interparticle interactions. ${ }^{12}$ For AuNSs-EPS, a second, more intense peak appeared at $760 \mathrm{~nm}$. AuNRs-EPS produces two new bands at 815 and $960 \mathrm{~nm}$. The AuNPrs-EPS exhibits a new broad band centered around $1100 \mathrm{~nm}$. These UV-vis spectra are consistent with theoretical predictions and experimental evidences for AuNP assemblies. ${ }^{12}$ The number of absorption peaks and their frequencies of gold aggregates depend on the degree of aggregation and the orientation of the individual particles within the aggregate. The first peak, located near the resonance peak for single nanoparticles, is attributed to the quadrupole plasmon excitation in coupled gold nanospheres. The second peak, at longer wavelengths, is attributed to the dipole plasmon resonance of the gold nanosphere. This absorption splitting is a consequence of the fact that oscillating electrons in one particle feel the electric field due to the oscillations in a second particle, which can lead to a collective plasmon oscillation of the gold aggregates. ${ }^{12}$

\section{B. Synthesis of gold nanoparticles using EPS as reductant (Au-EPS)}

In a second phase, we addressed the possibility of producing AuNPs aggregate by EPS and gold cations in one step. Carbohydrates in general and EPS in particular have been used as reducing and stabilizing agents for AuNPs formation. ${ }^{26-28}$

We used EPS of $L$. fermentum as a reducing agent by adding it to a boiling aqueous solution of $\mathrm{Au}(\mathrm{III})$. The resulting mixture (Au-EPS) was analyzed by TEM.

As shown in Fig. 3, AuNPs were formed on EPS. The contrast of the darker, electron-dense gold enhances the visualization of the lighter EPS material. A range of different sizes and shapes of 


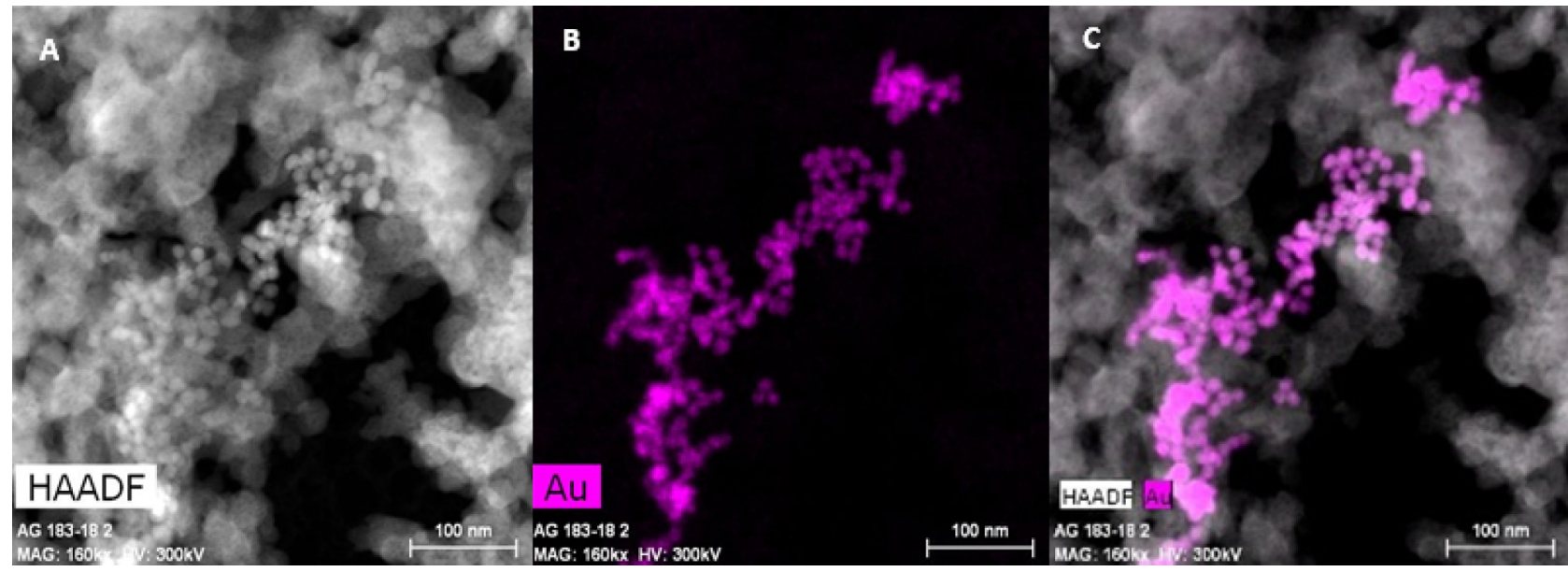

FIG. 2. (a) HAADF micrograph of AuNSs after incubation with EPS (AuNSs-EPS). (b) EDX compositional map of gold (violet) collected over the whole HAADF-STEM image in (a). (c) Compositional map of (a) and (b) images. Similar images were obtained for AuNRs-EPS and AuNPrs-EPS.

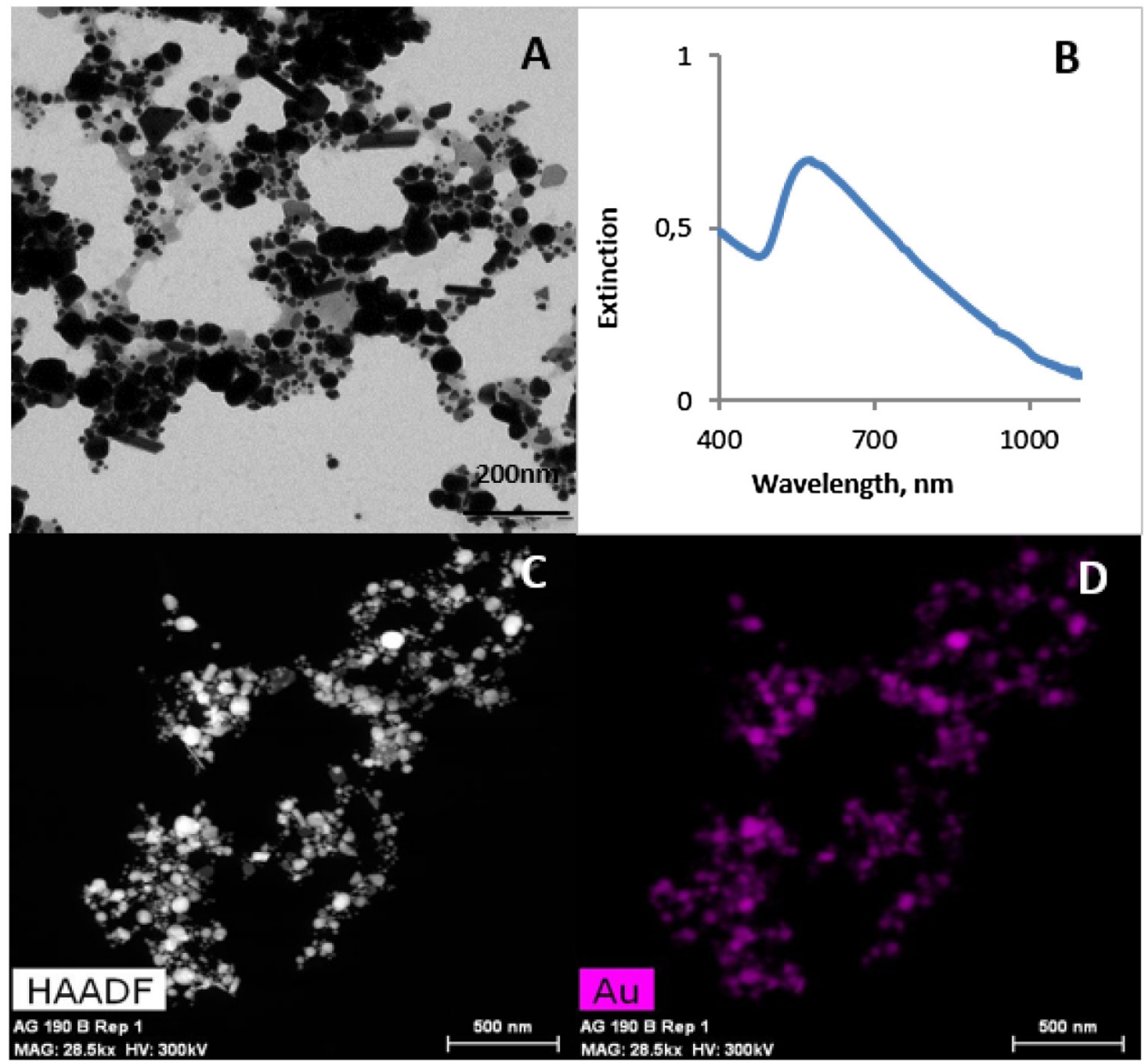

FIG. 3. (a) TEM image of Au-EPS and (b) its UV-vis spectrum. (c) HAADF micrograph of Au-EPS and (d) EDX compositional map of gold (violet) collected over the whole HAADF-STEM image in (c). 
nanoparticles were seen: spheres, rods, and prisms of different sizes $(2-80 \mathrm{~nm})$ are clearly noticeable from the TEM image.

The aggregates were visualized by HAADF-STEM (Fig. 3). To confirm the presence of the AuNPs on the EPS, energy dispersive X-ray spectroscopy (EDX) experiments were performed, which showed that Au (in violet) is linked to EPS [Figs. 3(c) and 3(d)]. The spatial distribution of gold, which was barely detectable outside the EPS region, demonstrates that the AuNPs were produced and incorporated onto the EPS. In these experimental conditions, EPS of L. fermentum produced heterogeneous gold particles without preferred size and shapes. In accordance with this result, the UV-vis spectrum of Au-EPS consists of a broad band with a peak maximum centered at $570 \mathrm{~nm}$ and a tail absorbing up to $1000 \mathrm{~nm}$ [Fig. 3(b)].

Since band position is dependent on both the number of coupled nanoparticles and their relative position with respect to each other and to the incident light, this broad band is the result of the contribution of differently shaped AuNPs and a lack of uniform distribution. It should be noted the lower absorbance values in the UV-visible spectrum of the sample Au-EPS [Fig. 3(b)] with respect to those of AuNSs-EPS, AuNRs-EPS, and AuNPrs-EPS (Fig. 1). The concentration of $\mathrm{Au}(\mathrm{III})$ incubated with EPS to form Au-EPS was the same $(0.25 \mathrm{mM})$ as that used in gold nanoparticle synthesis for the samples AuNSs-EPS, AuNRs-EPS, and AuNPrs-EPS. Therefore, the low absorbance values found for Au-EPS indicates that the EPS does not reduce all gold cations but just a fraction, which leads to a lower concentration of gold nanoparticles.

\section{SERS experiments}

We have applied these AuNPs materials to improve the SERS signals of an analyte. As shown by TEM, EPS efficiently glues AuNPs together. The short distances between AuNPs generate hot spots, where if an analyte deposits it, can improve their SERS signals. ${ }^{13-17}$

We tested the SERS performance of all gold aggregates for the detection of the model analyte rhodamine $\mathrm{B}(\mathrm{RhB})$. We analyzed the SERS performance of four samples: the three AuNPs morphologies deposited onto EPS (AuNSs, AuNRs, and AuNPrs) and the AuNPs made by the EPS themselves (Au-EPS). We incubated RhB

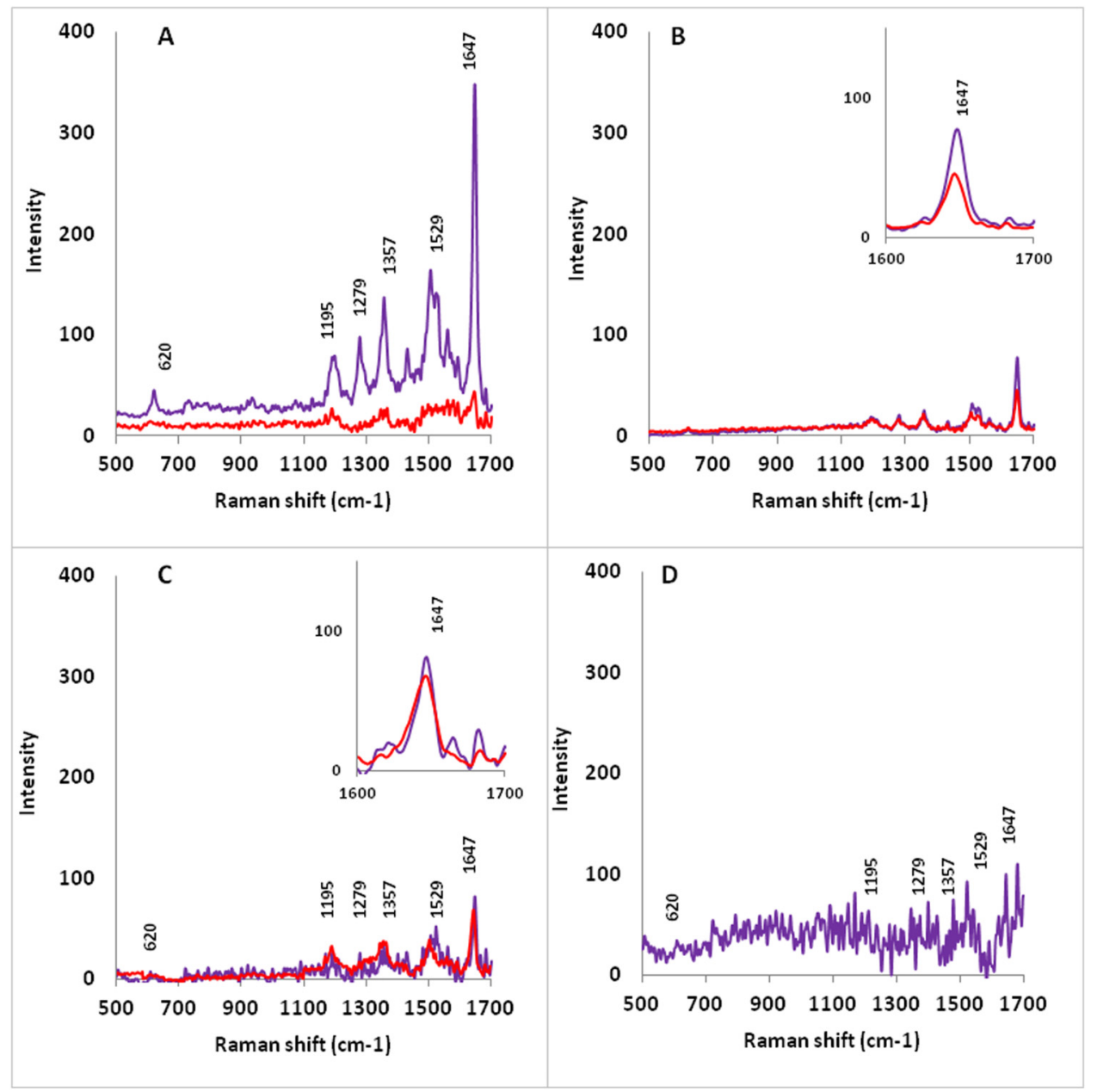

FIG. 4. Raman spectra of RhB (red) in the presence of isolated AuNPrs (a), AuNSs (b), and AuNRs (c). In violet are the RhB Raman spectra in the presence of aggregated samples: AuNPrs-EPS (a), AuNSs-EPS (b), AuNRs-EPS (c), and Au-EPS (d). 


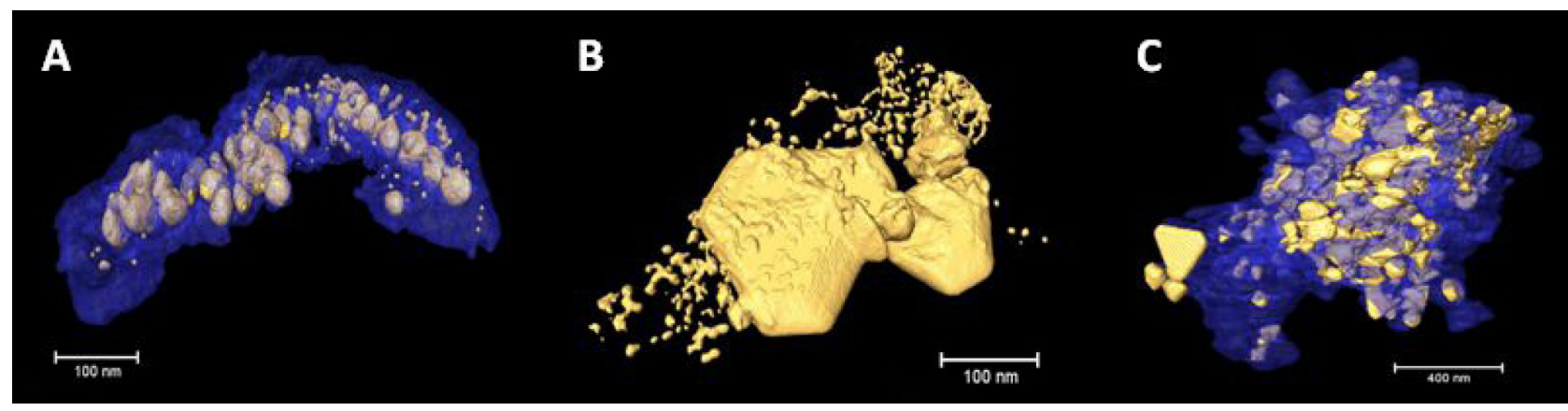

FIG. 5. Electron tomography results (rendered 3D volumes) of AuNSs (a), AuNPrs-EPS (b), and Au-EPS (c).

$(1 \mu \mathrm{M})$ with gold samples $(0.25 \mathrm{mM}$ in $\mathrm{Au})$ in water, and the mixtures were analyzed by SEM and Raman spectroscopy.

A total of 10 Raman spectra were collected on 10 different regions of the SEM grid. Representative Raman spectra obtained for each sample are shown in Fig. 4. SEM images of sample sections used for SERS are included (see Fig. S1 in the supplementary material).

Interestingly, the SERS results differed dramatically depending on the gold sample. A marginal increase of the Raman signal intensity of RhB was observed for the aggregated samples AuNSs-EPS and AuNRs-EPS with respect to the isolated samples, AuNSs and AuNRs, respectively [Figs. 4(b) and 4(c)]. However, the intensity of Raman signals of RhB in the presence of AuNPrs-EPS [Fig. 3(a), violet spectrum] was two orders of magnitude higher than that of AuNPrs [Fig. 4(a), red spectrum].

The dramatic increase in the Raman signal intensity of $\mathrm{RhB}$ in the presence of AuNPrs-EPS is not related to the prism shape of gold nanoparticles, since isolated AuNPrs did not exhibit a significant SERS effect [Fig. 4(a), red]. The increase does correspond to the aggregated state of the nanoparticles.

In order to understand the size of the SERS effect of AuNPrs-EPS in comparison to the rest of samples, we point out that although EPS glues the gold nanoparticles together, RhB access to the regions in between individual nanoparticles is not the same in all samples. As the SERS effect depends on RhB reaching the hot spots, this difference in accessibility results in the different SERS effects. To evaluate this hypothesis, we carried out a tomography study of all samples.

\section{Electron tomography (ET)}

This study revealed clear differences between the samples in terms of nanoparticle spatial distribution with respect to the EPS layer between the samples. As is evident from Fig. 5(a), in the AuNPSs-EPS sample, the gold appears in aggregates of spherical-like nanoparticles mostly embedded within EPS. Note that yellow and blue colors represent gold and EPS, respectively. Although some of these AuNPSs partially expose EPS-free surfaces [Fig. 5(a), very few yellow sections], most of them are located at positions where they are fully embedded in the EPS shell which averages a thickness of $24 \mathrm{~nm}$ (see Fig. S2 in the supplementary material).
The situation is different for AuNPrs-EPS, the sample that exhibits the highest SERS effect. In this case, groups of prismaticlike Au nanoparticles of largely varying size, and in close proximity to each other, depict mostly clean surfaces [Fig. 5(b)]. Although a very small number of polymer filamentary structures attached to the nanoparticles are visible, most of their surface remains free from EPS [the yellow color of gold; it is clearly predominant in Fig. 5(b)]. This spatial distribution of the two components favors the access of external molecules, such as RhB, to the hot spot locations between gold nanoprisms. This difference in exposed nanoparticle surface explains the drastic increase of intensity in the SERS spectrum and the improvement of the Raman spectrum of RhB with well-resolved and intense peaks. ${ }^{29}$ Finally, the Au-EPS sample showed a mixture of heterogeneously sized and shaped gold nanoparticles [Fig. 5(c)], which appear much more agglomerated than in AuNPrs-EPS. In contrast with the latter, particles are partially embedded within EPS, similar to what occurs in AuNPSs-EPS. A more compact EPS layer with an average thickness of $87 \mathrm{~nm}$ (see Fig. S3 in the supplementary material) in direct contact with a significant fraction of the Au nanoparticles is present in this sample. In this case, the EPS layer limits RhB access to the $\mathrm{Au}$ surfaces.

\section{CONCLUSIONS}

EPS of $L$. fermentum, a healthy bacteria present in our microbiota, is an extraordinarily effective bioplatform for aggregating gold nanoparticles of different sizes and shapes as well as for directly producing gold aggregates from a $\mathrm{Au}(\mathrm{III})$ solution. All gold aggregates were water-soluble and could be lyophilized and safely stored as a powder.

The gold aggregates on EPS improved the optical properties of isolated gold nanoparticles and showed additional absorptions at lower energies. SERS effects of gold aggregates were significantly different from isolated nanoparticles. An electronic tomography study confirmed that efficiency as a RhB SERS probe directly correlates with the extension of EPS coating of hot spots of gold aggregates. In particular, the sample AuNPrs-EPS, consisting of aggregated gold prisms onto the EPS, displayed $\mathrm{RhB}$ Raman spectra with intensities two orders of magnitude higher than free RhB. 


\section{SUPPLEMENTARY MATERIAL}

See the supplementary material for SEM images of sections analyzed by Raman spectroscopy (Fig. S1) and electron tomography thickness map of the EPS shell in the sample AuNSs-EPS (Fig. S2) and AUNPSs-EPS (Fig. S3).

\section{ACKNOWLEDGMENTS}

This work was funded by MINECO and FEDER (Project Nos. CTQ2015-64538-R and MAT2013-40823-R). Junta de Andalucia (Nos. FQM368 and FQM334) is also acknowledged. M. López-Haro acknowledges funding from Juan de la Cierva MINECO Program (Ref. No. IJCI-2014-19367). ET experiments were run at the Electron Microscopy Division (DME) of SC-ICYT UCA.

\section{REFERENCES}

${ }^{1}$ N. Li, P. Zhao, and D. Astruc, "Anisotropic gold nanoparticles: Synthesis, properties, applications, and toxicity," Angew. Chem. Int. Ed. 53(7), 1756-1789 (2014).

${ }^{2}$ K. Saha, S. S. Agasti, C. Kim, X. Li, and V. M. Rotello, "Gold nanoparticles in chemical and biological sensing," Chem. Rev. 112(5), 2739-2779 (2012).

${ }^{3}$ H. Jans and Q. Huo, "Gold nanoparticle-enabled biological and chemical detection and analysis," Chem. Soc. Rev. 41(7), 2849-2866 (2012).

${ }^{4}$ L. Dykman and N. Khlebtsov, "Gold nanoparticles in biomedical applications: Recent advances and perspectives," Chem. Soc. Rev. 41(6), 2256-2282 (2012).

${ }^{5}$ L. C. Kennedy, L. R. Bickford, N. A. Lewinski, A. J. Coughlin, Y. Hu, E. S. Day, J. L. West, and R. A. Drezek, "A new era for cancer treatment: Gold-nanoparticle-mediated thermal therapies,” Small 7(2), 169-183 (2011).

${ }^{6}$ R. Cao-Milán and L. M. Liz-Marzán, "Gold nanoparticle conjugates: Recent advances toward clinical applications," Expert Opin. Drug Deliv. 11(5), 741-752 (2014).

${ }^{7}$ N. S. Abadeer and C. J. Murphy, "Recent progress in cancer thermal therapy using gold nanoparticles," J. Phys. Chem. C 120(9), 4691-4716 (2016).

${ }^{8}$ J. Pérez-Juste, I. Pastoriza-Santos, L. M. Liz-Marzán, and P. Mulvaney, "Gold nanorods: Synthesis, characterization and applications," Coord. Chem. Rev. 249(17-18), 1870-1901 (2005).

${ }^{9}$ Y.-C. Yeh, B. Creran, and V. M. Rotello, "Gold nanoparticles: Preparation, properties, and applications in bionanotechnology," Nanoscale 4(6), 1871-1880 (2012).

${ }^{10}$ C. Carnovale, G. Bryant, R. Shukla, and V. Bansal, "Size, shape and surface chemistry of nano-gold dictate its cellular interactions, uptake and toxicity," Prog. Mater. Sci. 83, 152-190 (2016).

${ }^{11}$ J. Reguera, J. Langer, D. Jimenez de Aberasturi, and L. M. Liz-Marzan, "Anisotropic metal nanoparticles for surface enhanced Raman scattering," Chem. Soc. Rev. 46(13), 3866-3885 (2017).

${ }^{12}$ S. K. Ghosh and T. Pal, "Interparticle coupling effect on the surface plasmon resonance of gold nanoparticles: From theory to applications," Chem. Rev. 107(11), 4797-4862 (2007).

${ }^{13}$ D. Das, T. Kar, and P. K. Das, "Gel-nanocomposites: Materials with promising applications,” Soft Matter 8(8), 2348-2365 (2012).
${ }^{14}$ R. A. Alvarez-Puebla, A. Agarwal, P. Manna, B. P. Khanal, P. Aldeanueva-Potel, E. Carbó-Argibay, N. Pazos-Pérez, L. Vigderman, E. R. Zubarev, N. A. Kotov, and L. M. Liz-Marzán, “Gold nanorods 3D-supercrystals as surface enhanced Raman scattering spectroscopy substrates for the rapid detection of scrambled prions," Proc. Natl. Acad. Sci. U.S.A. 108(20), 8157-8161 (2011).

${ }^{15}$ N. Pazos-Perez, C. S. Wagner, J. M. Romo-Herrera, L. M. Liz-Marzán, F. J. García de Abajo, A. Wittemann, A. Fery, and R. A. Alvarez-Puebla, "Organized plasmonic clusters with high coordination number and extraordinary enhancement in surface-enhanced Raman scattering (SERS)," Angew. Chem. Int. Ed. 51(51), 12688-12693 (2012).

${ }^{16}$ J. Xu, L. Zhang, H. Gong, J. Í. Homola, and Q. Yu, “Tailoring plasmonic nanostructures for optimal SERS sensing of small molecules and large microorganisms," Small 7(3), 371-376 (2011).

${ }^{17}$ S. Barbosa, A. Agrawal, L. Rodríguez-Lorenzo, I. Pastoriza-Santos, R. A. Alvarez-Puebla, A. Kornowski, H. Weller, and L. M. Liz-Marzán, "Tuning size and sensing properties in colloidal gold nanostars," Langmuir 26(18), 14943-14950 (2010).

${ }^{18}$ J. S. Gunn, L. O. Bakaletz, and D. J. Wozniak, "What's on the outside matters: The role of the extracellular polymeric substance of gram-negative biofilms in evading host immunity and as a target for therapeutic intervention," J. Biol. Chem. 291(24), 12538-12546 (2016).

${ }^{19}$ E. Zannini, D. M. Waters, A. Coffey, and E. K. Arendt, "Production, properties, and industrial food application of lactic acid bacteria-derived exopolysaccharides," Appl. Microbiol. Biotechnol. 100(3), 1121-1135 (2016).

${ }^{20}$ A. K. Patel, P. Michaud, R. R. Singhania, C. R. Soccol, and A. Pandey, "Polysaccharides from probiotics: New developments as food additives," Food Technol. Biotechnol. 48(4), 451 (2010).

${ }^{21}$ S. V. N. Vijayendra, G. Palanivel, S. Mahadevamma, and R. N. Tharanathan, "Physico-chemical characterization of an exopolysaccharide produced by a non-ropy strain of Leuconostoc sp. CFR 2181 isolated from dahi, an Indian traditional lactic fermented milk product," Carbohydr. Polym. 72(2), 300-307 (2008). ${ }^{22}$ A. D. McFarland, C. L. Haynes, C. A. Mirkin, R. P. Van Duyne, and H. A. Godwin, “Color my nanoworld,” J. Chem. Educ. 81(4), 544A (2004).

${ }^{23} \mathrm{~B}$. Nikoobakht and M. A. El-Sayed, "Preparation and growth mechanism of gold nanorods (NRs) using seed-mediated growth method," Chem. Mater. 15, 1957 (2003).

${ }^{\mathbf{2 4}}$ Y. Jin, J. Deng, J. Liang, C. Shan, and M. Tong, "Efficient bacteria capture and inactivation by cetyltrimethylammonium bromide modified magnetic nanoparticles," Colloids Surf. 136, 659-665 (2015).

${ }^{25}$ B. Pelaz, V. Grazu, A. Ibarra, C. Magen, P. del Pino, and J. M. de la Fuente, "Tailoring the synthesis and heating ability of gold nanoprisms for bioapplications," Langmuir 28(24), 8965-8970 (2012).

${ }^{26}$ Pradeepa, S. M. Vidya, S. Mutalik, K. Udaya Bhat, P. Huilgol, and K. Avadhani, "Preparation of gold nanoparticles by novel bacterial exopolysaccharide for antibiotic delivery," Life Sci. 153, 171-179 (2016).

${ }^{27}$ G. Sathiyanarayanan, V. Vignesh, G. Saibaba, A. Vinothkanna, K. Dineshkumar, M. B. Viswanathan, and J. Selvin, "Synthesis of carbohydrate polymer encrusted gold nanoparticles using bacterial exopolysaccharide: A novel and greener approach," RSC Adv. 4(43), 22817-22827 (2014).

${ }^{28}$ S. K. Das, A. R. Das, and A. K. Guha, "Microbial synthesis of multishaped gold nanostructures," Small 6(9), 1012-1021 (2010).

${ }^{29}$ R. A. Alvarez-Puebla and L. M. Liz-Marzán, "SERS detection of small inorganic molecules and ions,” Angew. Chem. Int. Ed. 51(45), 11214-11223 (2012). 